\title{
Hiperglucemia inducida por glucocorticoides en el paciente hospitalizado: enfoque y manejo
}

\section{Hyperglycemia Induced by Glucocorticoids on Hospitalized Patient: Approach and Management}

\section{Rossanna Tous Lopera ${ }^{1}$, Andrés Felipe Coy Barrera ${ }^{1}$, William Rojas García ${ }^{2}$}

\author{
${ }^{1}$ Fellow Endocrinología Fundación Universitaria de Ciencias de la \\ Salud. \\ ${ }^{2}$ Profesor Titular Fundación Universitaria de Ciencias de la Salud, \\ Jefe de Servicio Endocrinología Hospital San José. \\ Fecha de recepción: 21/01/2019 \\ Fecha de aceptación: 05/02/2019
}

\section{Resumen}

La hiperglucemia inducida por glucocorticoides es un escenario frecuente al cual se enfrenta el clínico durante su práctica hospitalaria diaria, constituyendo un reto en el abordaje y control del estado metabólico de estos pacientes, con el fin de disminuir su morbimortalidad asociada. Presentamos el caso clínico de una paciente de género femenino de 69 años con antecedente de diabetes mellitus tipo 2 y linfoma no Hodgkin, hospitalizada para inicio de ciclo quimioterapéutico, durante el cual se documentan hiperglucemias sostenidas de difícil manejo. La paciente recibió tratamiento con esquema basalbolo y un bolo adicional de insulina NPH titulado según las dosis y tipo de glucocorticoide aplicado, alcanzando adecuado control metabólico.

Se realiza una revisión de la literatura respecto a la hiperglucemia inducida por glucocorticoides, su abordaje y tratamiento, y las recomendaciones existentes en cuanto al uso de insulina NPH como esquema adicional correctivo.

Palabras clave: hiperglucemia, glucocorticoide.

\section{Abstract}

Glucocorticoid-induced hyperglycemia is a frequent scenario faced by the clinician during his daily hospital practice, constituting a challenge in the approach and control of the metabolic state of these patients in order to reduce their associated morbidity and mortality. We present the clinical case of a 69-year-old female patient with a history of type 2 diabetes mellitus and non-hodgkin's lymphoma, hospitalized for the start of the retreatment cycle, during which sustained hyperglycemia of difficult management is documented. The patient received treatment with basal bolus protocol and an additional bolus of NPH insulin titrated according to the dose and type of glucocorticoid applied, achieving adequate metabolic control.

A review of the literature regarding glucocorticoid-induced hyperglycemia, its approach and treatment, and existing recommendations regarding the use of NPH insulin as an additional corrective scheme is made.

Keywords: hyperglycemia, glucocorticoids.

\section{Caso clínico}

Mujer de 69 años que ingresa por antecedente de linfoma no Hodgkin B difuso de células grandes con compromiso de amígdala izquierda, extensión a orofaringe y úvula, asociado a diabetes mellitus 2 e hipertensión arterial. Antes del ingreso, la paciente presenta laboratorios con $\mathrm{HbA} 1 \mathrm{c}$ de 8,2 y creatinina de $0,6 \mathrm{mg} / \mathrm{dl}$. Ingresa por servicio de hematología, se encuentra en esquema de quimioterapia RCHOP por lo que recibe prednisona $50 \mathrm{mg}$ vía oral durante cinco días, motivo por el cual se remite a interconsulta al servicio de endocrinología.

La paciente venía recibiendo de manera ambulatoria insulina glargina 12 UI cada noche asociada a 3 UI de insulina glulisina antes de cada comida, metformina $850 \mathrm{mg}$ vía oral/ día y atorvastatina $40 \mathrm{mg}$ vía oral/día.

Teniendo en cuenta el antecedente de diabetes mellitus asociada a dosis de glucocorticoide, se decide iniciar manejo con insulina NPH 20 UI, la cual se aplica al mismo tiempo que la dosis de glucocorticoide.

Las glucometrías de la paciente se presentan en la tabla 1 desde el primer día hasta el último día. La dosis de glucocorticoide se daba en la mañana.

Se presentan las glucometrías únicamente de ayunas, prealmuerzo y dos horas pos-almuerzo, teniendo en cuenta farmacodinamia del glucocorticoide.

En la actualidad la terapéutica con glucocorticoides incluye un $0,9 \%$ de la población general, aumentando hasta un $2,5 \%$ en personas entre 70 y 79 años y hasta un cuarto de esta 
Tabla 1.

\begin{tabular}{c|c|c|c}
\hline Día de esteroide & Ayunas & Pre-almuerzo & Dos horas pos-almuerzo \\
\hline 1 & $256 \mathrm{mg} / \mathrm{dl}$ & $198 \mathrm{mg} / \mathrm{dl}$ & $235 \mathrm{mg} / \mathrm{dl}$ \\
\hline 2 & $175 \mathrm{mg} / \mathrm{dl}$ & $210 \mathrm{mg} / \mathrm{dl}$ & $269 \mathrm{mg} / \mathrm{dl}$ \\
\hline 3 & $150 \mathrm{mg} / \mathrm{dl}$ & $194 \mathrm{mg} / \mathrm{dl}$ & $201 \mathrm{mg} / \mathrm{dl}$ \\
\hline 4 & $127 \mathrm{mg} / \mathrm{dl}$ & $186 \mathrm{mg} / \mathrm{dl}$ & $190 \mathrm{mg} / \mathrm{dl}$ \\
\hline
\end{tabular}

población los utilizan más allá de seis meses ${ }^{(1,2)}$. Los glucocorticoides son los medicamentos más frecuentemente asociados con el inicio de hiperglucemia ${ }^{(3)}$. Exacerban la hiperglucemia en pacientes diabéticos o precipitan la aparición de la diabetes por glucocorticoides y se convierten en un factor de riesgo independiente para otras complicaciones asociadas a su uso ${ }^{(4)}$ tales como deshidratación, inmunosupresión con aumento de las infecciones, estados hiperosmolares o cetoacidosis diabética y se asocian con mayor mortalidad ${ }^{(5)}$.

Frecuentemente los pacientes hospitalizados deben recibir glucocorticoides, siendo los medicamentos más frecuentemente relacionados con hiperglucemias intrahospitalarias ${ }^{(1)}$. En un estudio, 50\% de los pacientes tratados con glucocorticoides tuvieron glucemias mayores de $200 \mathrm{mg} / \mathrm{dl}^{(6)}$. Dentro de las indicaciones más frecuentes de uso de glucocorticoides en pacientes hospitalizados se encuentran las de tratamiento de enfermedades reumatológicas como artritis reumatoide y lupus, de causa nefrológica como glomerulonefritis y vasculitis, por enfermedades gastrointestinales, enfermedad inflamatoria intestinal, hepatitis autoinmune, entre otras; de causa hematológica como linfoma o mieloma múltiple, en infecciones como meningitis o choque séptico, en enfermedades neumológicas como EPOC y asma, en reacciones alérgicas ${ }^{(7)}$ y en la actualidad frecuentemente en posoperatorios para disminuir las náuseas y vómitos inducidos por anestésicos ${ }^{(8)} \mathrm{y}$ en pacientes trasplantados ${ }^{(10)}$, incluyendo trasplante de páncreas ${ }^{(10)}$.

El pronóstico intrahospitalario se afecta negativamente en pacientes hiperglucémicos, llegando casi a duplicar la mortalidad $^{(11)}$.

Desde la primera descripción del incremento de la glucemia en pacientes tratados con glucocorticoides ${ }^{(12)}$, mucho se han estudiado los múltiples mecanismos fisiopatológicos implicados, siendo los más claros el incremento de la resistencia periférica a la insulina con aumento de la producción y secreción de insulina por las células $\beta$ del páncreas. Por otra parte, los glucocorticoides incrementan la producción de glucosa mediante la activación de múltiples genes que producen gluconeogénesis ${ }^{(13,14)}$. Producen también la inhibición de la captación periférica de la glucosa por el músculo estriado ${ }^{(15)}$.

El equilibrio sistémico de la glucosa se mantiene permanentemente por la regulación de la producción endógena de glucosa y su utilización a nivel periférico ${ }^{(16)}$. La producción de glucosa se realiza por gluconeogénesis o glucogenólisis princi- palmente en el hígado y en menor grado por los riñones ${ }^{(17)}$. La gluconeogénesis resulta de la conversión de precursores nocarbohidratos como el lactato, la alanina y el glicerol a glucosa en el hígado ${ }^{(18)}$. El exceso de glucosa se polimeriza a glucógeno y se almacena principalmente en el hígado y el músculo. La hiperglucemia se desarrolla debido a tres procesos: 1 . Aumento de la gluconeogénesis. 2. Glucogenólisis acelerada y 3. Deterioro de la utilización de la glucosa por los tejidos periféricos. Desde el punto de vista cuantitativo, la mayor producción de glucosa hepática representa el mayor impacto en las cifras de glucosa en sangre. El aumento de la producción de glucosa hepática resulta de la alta disponibilidad de precursores gluconeogénicos como los aminoácidos principalmente alanina y glutamina, produciéndose una proteólisis acelerada y una disminución de la síntesis proteínica, que aumenta el lactato y resulta en un incremento de la glucogenólisis muscular con producción de glicerol por la lipólisis creciente, aumentando la actividad de las enzimas gluconeogénicas como fosfoenolpiruvato carboxicinasa, la fructosa-1,6-bifosfatasa y la piruvato carboxilasa. El metabolismo de la glucosa es mantenido por una interacción de las hormonas reguladoras, principalmente la insulina, y por las hormonas contrarreguladoras como el glucagón, el cortisol, la hormona de crecimiento y las catecolaminas. La insulina controla la producción de glucosa hepática, suprimiendo la gluconeogénesis hepática y la glucogenólisis. En los tejidos sensibles a la insulina como el músculo, la insulina promueve el anabolismo de proteínas, la toma de glucosa y la síntesis de glucógeno e inhibe la glucogenólisis y la ruptura proteínica. Además, la insulina es un gran inhibidor de la lipólisis, a oxidación libre de ácidos grasos y de la cetogénesis ${ }^{(19)}$.

El glucagón es la hormona glucogenolítica más importante, actúa regulando la producción de glucosa hepática durante el estado normal y en estados de hiperglucemia. Durante el estrés, el exceso de concentración de hormonas contrarreguladoras induce resistencia a la insulina, aumenta la producción de glucosa hepática y reduce la utilización de la glucosa periférica. Los altos niveles de epinefrina estimulan la secreción de glucagón e inhiben la liberación de insulina por las células $\beta$ pancreáticas ${ }^{(20)}$.

El desarrollo de la hiperglucemia da como resultado un estado inflamatorio caracterizado por una elevación de citocinas proinflamatorias y aumento de los marcadores de estrés oxidativo $^{(21,22,23)}$. En pacientes con hiperglucemia grave se incrementan sustancialmente los niveles séricos de factor de 
Figura 1. Patogénesis de la hiperglucemia

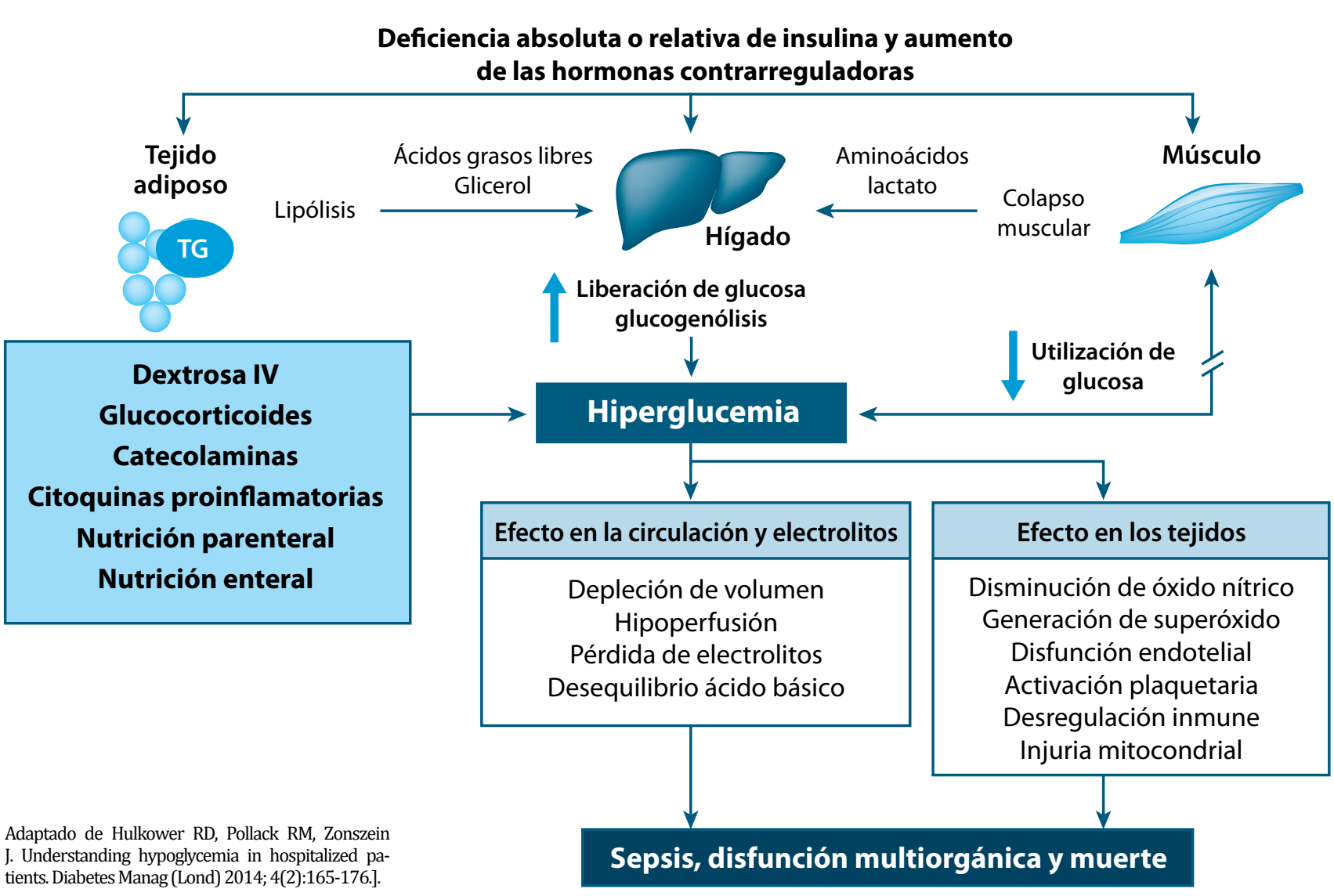

necrosis tumoral- $\alpha$, interleucinas [IL]-6, IL1- $\beta$ e IL-8, proteína $\mathrm{C}$-reactiva los cuales regresan a niveles basales con el restablecimiento de los niveles normales de glucosa con insulina. El TNF- $\alpha$ produce resistencia a la insulina por su interacción con receptor de la insulina. Durante el estrés agudo, las citocinas inflamatorias aumentan la resistencia a la insulina y el TNF- $\alpha$ bloquea la activación insulina-mediada por la fosfatidilinositol 3 cinasa, reduciendo la captación de la insulina en tejidos periféricos $^{(24,25)}$ (figura 1).

Para un abordaje estandarizado del paciente hiperglicémicos por glucocorticoides empleamos la tabla de equivalencias de la potencia de los glucocorticoides para ajustar todos los algoritmos con base en esta equivalencia (tabla 2) Y sugerimos tomar de base las definiciones de la "Asociación Americana de Diabetes"(26).

Definitivamente, tanto la hiperglucemia como la hipoglucemia se asocian con peor pronóstico en pacientes hospitalizados, por lo cual es esencial promover estancias cortas y seguras y una adecuada transición al ambiente ambulatorio, previniendo complicaciones y readmisiones. La primera recomendación es realizar una medición de la hemoglobina glucosilada en todos los pacientes con diabetes o hiperglucemia (glucosa en sangre $>$ de $140 \mathrm{mg} / \mathrm{dl}$ ) al ingreso al hospital si ésta no se ha realizado en los tres meses previos (grado B). El ideal es tener implementados protocolos que aseguren un cuidado de alta calidad para pacientes con diabetes ${ }^{(27)}$ y aun si es posible vale también la pena implementar unas órdenes médicas sistematizadas para aplicar desde el ingreso de estos pacientes. Una vez hospitalizado el paciente, se debe iniciar su educación o refuerzo de sus conductas para desarrollar o afianzar las habilidades necesarias para asegurar su correcto tratamiento al alta. La insulina debe ser administrada mediante un esquema predefinido que permita los ajustes en las dosis de acuerdo con las fluctuaciones de la glucemia durante la hospitalización (grado E). Esta recomendación previene errores e incrementa la eficiencia de la administración de medicaTabla 2. Equivalencia de glucocorticoides

\begin{tabular}{c|c|c|c} 
Dexametasona & Prednisolona & Metilprednisolona & Hidrocortisona \\
\hline $0.75 \mathrm{mg}$ & $5 \mathrm{mg}$ & $4 \mathrm{mg}$ & $20 \mathrm{mg}$ \\
\hline
\end{tabular}

Adaptado de Brady V, Grimes D, Amstrong T; et al. Management of steroid-induced hyperglycemia in Hospitalized patients with cancer review. Oncology Nursing Forum 2014(41) mentos $^{(28)}$. Especialistas o equipos tancia hospitalaria mejorando el control de la glucemia y mejorando el pronóstico de los pacientes ${ }^{(29)}$. El tratamiento de la hiperglucemia entrenados pueden reducir la es- 
Tabla 3. Fortalezas y debilidades de los hipoglucemiantes orales en el tratamiento de la hiperglucemia inducida por glucocorticoides

\begin{tabular}{|c|c|c|}
\hline Medicamento & Beneficios & Riesgos \\
\hline \multirow{3}{*}{ Sulfonilureas } & $\begin{array}{l}\text { Aumento de la } \\
\text { secreción de } \\
\text { insulina }\end{array}$ & $\begin{array}{c}\text { Pobre respuesta } \\
\text { (aproximadamente } \\
\text { 25\%) }\end{array}$ \\
\hline & $\begin{array}{c}\text { Rápido inicio de } \\
\text { acción }\end{array}$ & $\begin{array}{l}\text { Dificultades para } \\
\text { su titulación }\end{array}$ \\
\hline & Bajo costo & $\begin{array}{l}\text { Hipoglucemia con } \\
\text { cambio de dosis de } \\
\text { corticoides }\end{array}$ \\
\hline \multirow[t]{2}{*}{ Metformina } & $\begin{array}{l}\text { Aumento de la } \\
\text { secreción de } \\
\text { insulina }\end{array}$ & $\begin{array}{l}\text { Contraindicado } \\
\text { relativamente } \\
\text { en indicaciones } \\
\text { frecuentes }\end{array}$ \\
\hline & Bajo costo & $\begin{array}{c}\text { Pocos ensayos } \\
\text { clínicos }\end{array}$ \\
\hline \multirow{4}{*}{ Tiazolidinedionas } & $\begin{array}{l}\text { Aumento de la } \\
\text { secreción de } \\
\text { insulina }\end{array}$ & Edemas \\
\hline & $\begin{array}{l}\text { Alta respuesta } \\
\text { en terapias } \\
\text { combinadas }\end{array}$ & $\begin{array}{c}\text { Falla cardiaca } \\
\text { congestiva }\end{array}$ \\
\hline & & $\begin{array}{c}\text { Riesgos } \\
\text { cardiovasculares } \\
\text { posibles }\end{array}$ \\
\hline & & $\begin{array}{c}\text { Fracturas a largo } \\
\text { plazo }\end{array}$ \\
\hline
\end{tabular}

Modificado de Clore J, Thurby-Hay L. Glucocorticoid-induced hyperglucemia. Endocr Pract 2009; 15:472

debe comenzar con valores persistentes por encima de $180 \mathrm{mg} /$ dl. Una vez se inicia el esquema de insulina, la glucemia debe mantenerse entre $140-180 \mathrm{mg} / \mathrm{dl}$ tanto en pacientes críticos como en no críticos. En pacientes seleccionados, puede intentarse mantener valores entre 110 y $140 \mathrm{mg} / \mathrm{dl}$ sin hipoglucemias significativas (grado C). Se consideran valores de $<$ de $70 \mathrm{mg} / \mathrm{dl}$ alarmas de hipoglucemia y son significativos por debajo de 54 $\mathrm{mg} / \mathrm{dl}$. Hipoglucemia severa es aquella asociada con trastornos de conciencia, independiente del nivel sanguíneo ${ }^{(30)}$. El monitoreo con glucometrías debe ser realizado antes de las comidas. En pacientes sin vía oral entre cada 4 y 6 horas. Si el paciente tiene goteo endovenoso de insulina, puede requerir monitoreos desde cada 30 minutos hasta cada 2 horas ${ }^{(27)}$. Las recomendaciones de agentes antihiperglucemiantes en pacientes hospitalizados indican un régimen basal de corrección en bolo con insulina adicional en pacientes con adecuada ingesta de alimentos (grado A). Se desaconseja el uso exclusivo de un esquema móvil de insulina en estos pacientes (grado A). Si bien es cierto que los hipoglucemiantes orales se han empleado para el control de la hiperglucemia inducida por glucocorticoides, su uso tiene limitaciones ambulatorias $^{(31)}$ y en el contexto de la hiperglucemia hospitalaria su uso es aún más restringido tanto en pacientes con o sin glucocorticoides, debido a su estrecho margen terapéutico ${ }^{(32)}$. Los hipoglucemiantes orales se reservan solo para pacientes con valores de glucemia menor de $200 \mathrm{mg} / \mathrm{dl}$, previamente no diabéticos o diabéticos controlados solo con medidas no farmacológicas y preferiblemente se deben usar los iDPP4 por su efecto dependiente de la glucemia posprandial que se asocia a un bajo riesgo de hipoglucemia ${ }^{(1)}$. En la tabla 3 se describen en general las ventajas y los riesgos de los hipoglucemiantes orales. En los pacientes hospitalizados existen dos estrategias para el control de la hiperglucemia, sabiendo que cuando ésta es inducida por glucocorticoides su valor en ayunas es usualmente normal y el tratamiento se aplica en una sola dosis que además suele ser en las mañanas. La primera estrategia es la del uso de bolo de insulina preprandial de acuerdo con la elevación de glucemia después de la comida, la cual tiende a elevarse típicamente en la tarde o al anochecer ${ }^{(33)}$, descendiendo gradualmente durante el transcurso de la noche ${ }^{(34)}$. La segunda estrategia se basa en ajustar la insulina según el peso del paciente y la dosis de glucocorticoides administrada. Con índices de masa corporal mayores de 26 $\mathrm{kg} / \mathrm{m}^{2}$, existe un aumento lineal de la resistencia a la insulina ${ }^{(35)}$. Esto conlleva a usar una dosis basada en el cálculo de una dosis adicional media de 0,4 unidades/kg con una insulina basal como la NPH en pacientes con glucocorticoides de acción rápida como la prednisona y prednisolona (tabla 4).

Tabla 4. Resumen del protocolo de insulina en pacientes con glucocorticoides intrahospitalarios fuera de UCI

\begin{tabular}{|c|c|c|c|}
\hline & \multicolumn{3}{|c|}{ HbA1c } \\
\hline & $<6,5 \%$ & $6,5 \%-8,5 \%$ & $>8,5 \%$ \\
\hline $\begin{array}{l}\text { Sin diabetes o / } \\
\text { no diagnosticada }\end{array}$ & $\begin{array}{l}\text { Dosis de corrección de insulina con el } \\
\text { glucocorticoide/NA. }\end{array}$ & $\begin{array}{l}\mathrm{NA} / \text { iniciar dosis de corrección con } 0,4 \\
\mathrm{u} / \mathrm{kg}+\text { dosis de corrección con el glu- } \\
\text { cocorticoide. }\end{array}$ & $\begin{array}{l}\mathrm{NA} / \text { iniciar dosis de corrección con } 0,5 \\
\mathrm{u} / \mathrm{kg}+\text { dosis de corrección con el glu- } \\
\text { cocorticoide. }\end{array}$ \\
\hline $\begin{array}{l}\text { Diabético sin } \\
\text { bolo basal de } \\
\text { insulina }\end{array}$ & $\begin{array}{l}\text { Suspender medicación previa/insuli- } \\
\text { na de carga } 0,3 \mathrm{u} / \mathrm{k}+\text { dosis de correc- } \\
\text { ción con el glucocorticoide. }\end{array}$ & $\begin{array}{l}\text { Suspender medicación previa/iniciar } \\
\text { dosis de corrección con } 0,4 \mathrm{u} / \mathrm{kg}+\text { dosis } \\
\text { de corrección con el glucocorticoide. }\end{array}$ & $\begin{array}{l}\text { Suspender medicación previa/iniciar } \\
\text { dosis de corrección con } 0,5 \mathrm{u} / \mathrm{kg}+\text { dosis } \\
\text { de corrección con el glucocorticoide. }\end{array}$ \\
\hline $\begin{array}{l}\text { Diabético con } \\
\text { bolo basal de } \\
\text { insulina }\end{array}$ & $\begin{array}{l}\text { Continuar el bolo basal de insulina que } \\
\text { trae el paciente + dosis de corrección } \\
\text { de insulina con el glucocorticoide. }\end{array}$ & $\begin{array}{l}\text { Continuar el bolo basal de insulina que } \\
\text { trae el paciente + dosis de corrección de } \\
\text { insulina con el glucocorticoide. }\end{array}$ & $\begin{array}{l}\text { Continuar el bolo basal de insulina que } \\
\text { trae el paciente + dosis de corrección de } \\
\text { insulina con el glucocorticoide. }\end{array}$ \\
\hline
\end{tabular}

HbA1c Hemoglobina glucosilada. UCI Unidad de Cuidados Intensivos. NA: No aplicable 
Con un pico de acción entre 4 y 8 horas esta insulina tiene un pico intermedio de acción que refleja este comportamiento y además tiene una acción que dura entre 12 y 16 horas. Este esquema dosis-respuesta se basa en el conocimiento de la sensibilidad de los glucocorticoides a la insulina (tabla 5). En caso de glucocorticoides de acción prolongada como la dexametasona en la que se observan elevaciones de la glucosa hasta 20 horas después de su administración o en caso de dosis múltiples o uso continuo, las insulinas de larga acción como glargina o detemir pueden usarse (tabla 6). Se recomienda ajustar diariamente la dosis de insulina basal de acuerdo con la dosis total diaria usada las 24 horas previas y según los valores de glucemia. En el caso de aumentos de las dosis de glucocorticoides, el incremento de las dosis preprandiales o el uso de insulina suplementaria puede ser lo más apropiado, así como el aumento del bolo de insulina basal. Puede adicionarse entre el 50\% y el 100\% de insulina por bolo en caso de ser necesario. Por otro lado, se deben anticipar los cambios en las dosis de glucocorticoides, siendo lo más frecuente la disminución programada de las mismas, siendo en todos los casos crucial el monitoreo con glucometrías ${ }^{(36-39)}$.

La propuesta de administrar una dosis de insulina correctiva adicional que coincida con el perfil glucémico del glucocorticoide administrado busca anular el efecto hiperglucémico del glucocorticoide, ajustando al perfil farmacodinámico de éste y la insulina que se va a emplear. El primer paso consiste en clasificar el paciente como diabético previo o diabético de novo ${ }^{(40,41)}$. Los pacientes con diabetes mellitus se identifican ya sea porque consta en la historia clínica que tienen el antecedente de diabetes mellitus o porque en sus antecedentes han recibido medicamentos antidiabéticos orales o inyectables o insulina o porque su hemoglobina glucosilada (HbA1c) es igual o mayor a 6,5\%. El otro grupo de pacientes es el de quienes no se ha establecido el diagnóstico de diabetes mellitus, pero desarrollaron hiperglucemia después de la administración de glucocorticoides. El esquema de tratamiento es el uso de una dosis correctiva de insulina, la cual se administraría al mismo momento de la aplicación del glucocorticoide, de acuerdo con la dosis y el tipo de glucocorticoide administrado. Esto se presenta en las tablas 7 y 8 . Además de la dosis de corrección de insulina, los pacientes con diabetes mellitus establecida deben continuar con esquema basal-bolo y quienes no tienen diabetes no reciben ninguna insulina adicional. La indicación
Tabla 5. Dosis sugerida de insulina NPH de acuerdo con la dosis de glucocorticoides

\begin{tabular}{c|c}
\hline Prednisona (mg/dl) & Dosis de insulina (U/kg) \\
\hline$>40$ & 0,4 \\
\hline 30 & 0,3 \\
\hline 20 & 0,2 \\
\hline 10 & 0,1 \\
\hline
\end{tabular}

Modificada de Clore J, Thurby-Hay L. Glucocorticoid-induced hyperglucemia Endocr Pract 2009; 15:472.

Tabla 6.

\begin{tabular}{l|c|c|}
\hline \multicolumn{3}{|l}{ Farmacodinamia de los glucocorticoides } \\
\hline & $\begin{array}{c}\text { Prednisolona/ } \\
\text { Metilprednisolona }\end{array}$ & Dexametasona \\
\hline $\begin{array}{l}\text { Inicio de efectos } \\
\text { hipoglucémicos }\end{array}$ & 4 horas & 4 horas \\
\hline Pico & 8 horas & Desconocido \\
\hline Resolución & $12-16$ horas & $24-36$ horas \\
\hline Farmacodinamia de las insulinas & \\
\hline Inicio & NPH & Glargina \\
\hline Pico & $1-2$ horas & $1-6$ horas \\
\hline Resolución & $3-8$ horas & Sin pico \\
\hline
\end{tabular}

Modificado de Wallace M, Metzger N. Optimizing the treatment of Steroid Induced Hyperglycemia. Annals of Pharmacoterapy, 2017.

Tabla 7. Tipo de insulina correctiva que se debe usar según el tipo de glucocorticoide administrado

\begin{tabular}{l|l}
\hline \multicolumn{1}{c|}{ Tipo de insulina } & \multicolumn{1}{c}{ Glucocorticoide } \\
\hline Insulina humana regular & Hidrocortisona \\
\hline Insulina NPH (Neutral & Prednisolona \\
Protamine Hagadorn) & Metilprednisolona \\
\hline Insulina glargina & Dexametasona \\
\hline
\end{tabular}

Modificado de Lhakani OJ, Kumar S, Triphati S, et al. Comparison of two protocols in the management of glucocorticoid-induced hyperglycemia among hospitalized patients. Indian Journal of Endocrinology and Metabolism. 2017;21,6:836-844.

Tabla 8. Dosis de corrección de insulina de acuerdo con el respectivo glucocorticoide

\begin{tabular}{c|c|c|c|c}
\hline Hidrocortisona (mg) & Prednisolona (mg) & Metilprednisolona (mg) & Dexametasona (mg) & Insulina (unidades/k) \\
\hline 50 & 10 & 8 & 2 & 0,1 \\
\hline 100 & 20 & 16 & 4 & 0,2 \\
\hline 150 & 30 & 24 & 6 & 0,3 \\
\hline 200 & $>40$ & 32 & 8 & 0,4 \\
\hline
\end{tabular}


de la insulina basal se detalla en la tabla $\mathbf{9}^{(4)}$. Los pacientes diabéticos reciben una dosis basal inicial del $50 \%$ basal y $50 \%$ de insulina la reciben en bolo ${ }^{(5)}$. Las guías indican que se debe iniciar insulina entre 0,3 y 0,5 unidades $/ \mathrm{kg}$ de peso (tabla 10) pero se pueden incluso llegar a emplear hasta 0,7 unidades $/ \mathrm{kg}$ de peso. La insulina basal usada es la insulina de larga acción que recibe el paciente diabético antes de hospitalizarse y quienes no presentan un esquema de este tipo o en los pacientes diabéticos de novo se recomienda insulina glargina de larga duración e insulina de corta acción, lispro, como bolo. Si se usa insulina glargina basal, ésta se administra a la hora de acostarse y la dosis se titula como se muestra la tabla 11. La insulina en bolo se administra 15 minutos antes de cada comida. La dosis suplementaria de la insulina de corta acción (lispro) se calcula de acuerdo con las glucometrías preprandiales. Las lecturas de las glucometrías antes de las comidas deben informarse al grupo de seguimiento de estos pacientes (grupo o médico) quien indica la dosis (tabla 11) con base en la escala suplementaria descrita en las pautas de la Sociedad de Endocrinología (tabla 4) ${ }^{(42)}$.

En pacientes con hiperglucemias persistentemente mayores de $300 \mathrm{mg} / \mathrm{dl}$ se deben usar esquemas más agresivos como infusión endovenosa de insulina o una dosis mayor en bolo-basal o correcciones más frecuentes con insulinas de acción rápida ${ }^{(42)}$.

En pacientes con dosis elevadas de glucocorticoides generalmente en el contexto de enfermedades oncológicas ${ }^{(43-46)}$, que alcanzan más de $250 \mathrm{mg} / \mathrm{dl}$ de glucometrías en más de dos ocasiones sin diabetes tipo I o falla renal (creatinina $>$ de $2 \mathrm{mg} / \mathrm{dl}$ ), se logró disminuir los niveles de glucemia sin llegar a niveles clínicamente aceptables pero sin aumentar significativamente los episodios de hipoglucemia y sin ninguna hipoglucemia severa. Además se encontró que a más ciclos de quimioterapia se requería más dosis de insulina para controlar la hiperglucemia, que aunque no fue estadísticamente significativa, sí se sugiere un aumento de la resistencia a la insulina en estos pacientes (figura 2). Dentro de las sugerencias resalta, en pacientes con anorexia, la aplicación de insulina solamente si el paciente consume más del $50 \%$ de las comidas inmediatamente al terminar éstas y realizar diariamente la revisión de los valores de las glucometrías y ajustar las dosis de insulina de acuerdo con la tendencia de éstos y usualmente hacer el ajuste en los bolos preprandiales y solamente aumentar las dosis del bolo basal si aumenta el nivel de glucemia nocturna y/o si hay hiperglucemia entre las comidas. Se observó también que los

Tabla 9. Dosis recomendada de insulina basal

\begin{tabular}{c|c}
\hline Glucometría en ayunas & $\begin{array}{c}\text { Cambio de la dosis basal de } \\
\text { insulina }\end{array}$ \\
\hline$>200 \mathrm{mg} / \mathrm{dl}$ & Aumentar el bolo basal $20 \%$ \\
\hline $140-199 \mathrm{mg} / \mathrm{dl}$ & Aumentar el bolo basal 10\% \\
\hline $70-100 \mathrm{mg} / \mathrm{dl}$ & Disminuir el bolo basal $10 \%$ \\
\hline$<70 \mathrm{mg} / \mathrm{dl}$ & Disminuir el bolo basal $20 \%$ \\
\hline
\end{tabular}

Tabla 10. Dosis total recomendada de inicio de insulina

\begin{tabular}{c|c} 
HbA1c (\%) & Dosis total de inicio U/kg \\
\hline$<6,5$ & 0,3 \\
\hline $6,5-8,5$ & 0,4 \\
\hline$>8,5$ & 0,5 \\
\hline $\begin{array}{c}>\text { de } 70 \text { años independiente } \\
\text { de HbA1c }\end{array}$ & 0,3 \\
\hline
\end{tabular}

HbA1c: Hemoglobina glucosilada

Tabla 11. Dosis suplementaria de insulina preprandial

\begin{tabular}{c|c}
\hline $\begin{array}{c}\text { Glucosa en sangre } \\
\text { (mg/dl) }\end{array}$ & $\begin{array}{c}\text { Dosis suplementaria de insulina } \\
\text { (U) }\end{array}$ \\
$>141-180$ & 4 \\
\hline $181-220$ & 6 \\
\hline $221-260$ & $\begin{array}{c}\text { 8 preprandial y si glucemia }>\text { de } 250 \\
\text { descartar cetoacidosis }\end{array}$ \\
\hline $261-300$ & 10 \\
\hline $301-350$ & 12 \\
\hline $351-400$ & 14 \\
\hline$>400$ & Avisar al grupo encargado según \\
protocolo institucional
\end{tabular}

\section{Figura 2.}

Criterios de inclusión:
Paciente con altas dosis de
glucocorticoide
Definidos como:
- Dexametasona $\geq 40 \mathrm{mg}$ diarios
- Metilprednisolona $\geq 250 \mathrm{mg}$
diarios
- Prednisona $\geq 300 \mathrm{mg}$ diarios

- Si el paciente recibe hipoglicemiante oral, continuar la terapia, si no existen contraindicaciones específicas.

- Si el paciente NO recibe hipoglucemiantes orales, considerar adicionar metformina, si no hay contraindicaciones específicas.

- Si el paciente recibe terapia con insulina, aumentar la dosis en 1-1,2 U/kg, distribuidos $25 \%$ basal y $75 \%$ prandial (distribuido en tres comidas).

- Si el paciente no recibe insulina, iniciar múltiples dosis de insulina a 1-1,2 U/kg, distribuidos en $25 \%$ basal y $75 \%$ prandial (distribuido en tres comidas).
Después de inicio de régimen de insulina, revalorar diariamente, considerando dosis correctivas de insulina si hay circunstancias especiales (dosis adicionales de glucocorticoide, comidas muy ricas en carbohidratos). A pesar de esto, si los valores $\geq 50 \%$ de la glucosa son: $\cdot \geq 200$ : aumentar la dosis total diaria un $20 \%$ - $\geq 300$ : aumentar la dosis total diaria un $30 \%$ $\cdot \geq 400$ : aumentar la dosis total diaria un $50 \%$

Modificada de Brady V, Thosani S, Zhou S, Bassett R, Busaidy NL, Lavis V. Safe and effective dosing of basal-bolus insulin in patients receiving high-dose steroids for hyper-cyclophosphamide, doxorubicin, vincristine, and dexamethasone chemotherapy. Diabetes Technol Ther 2014; 16: 874-879. 
pacientes previamente diabéticos tenían un mayor índice de masa corporal y requerían una dosis mayor de insulina basal y una mayor dosis de insulina diaria. La recomendación final en estos pacientes es la de aplicar un bolo basal del $25 \%$ del requerimiento de insulina y distribuir antes de cada comida el $75 \%$ restante, asegurando un monitoreo cercano para evi- tar hipoglucemia y de todas maneras se reconoce el estrecho margen para evitar hipoglucemias ${ }^{(47)}$. Estos esquemas son en el momento los métodos más precisos de control de la hiperglucemia con la menor cantidad de eventos de hipoglucemia y que permiten un adecuado control de los pacientes con hiperglucemia inducida por glucocorticoides.

\section{Referencias}

1. Perez A, Jansen-Chaparro S, Saigi I, Bernal-Lopez MR, Miñambres I, GomezHuelgas R. Glucocorticoid-induced hyperglycemia. Journal of Diabetes 6 (2014) 9-20.

2. Van Staa TP, Leufkens HGM, Abenhaim L, Begaud B, Zhang B, Cooper C. Use or oral corticosteroids in the United Kingdom. QJ Med. 2000; 93:105-11.

3. Bressler P, De Fonzo RA. Drugs and diabtes. Diabetes Rev.1994; 2: 53-84.

4. Tatsuno I, Sugiyama T. Glucocorticoides-induced diabetes mellitus is a risk for vertebral fracture during glucocorticoid treatment. Diabetes Res Clin Pract. 2011; 93: e18-e20

5. Rowbottom, Leigha, Stinson, Jordan, McDonlad, Rachel, et al. Retrospective review of the incidence of onitoring blood glucose levels in patients receiving corticosteroids with systemic anticancer therapy. Ann Palliat Med 2015:44(2); 70-77.

6. Donihi AC, Raval D, Saul M, Korykowski MT, De Vita MA. Prevalence and predictors of corticosteroids-related hyperglycemia in hospitalized patients. Endocr Pract. 2006;12: 358-62.

7. Raalte DH, Diamant M. Steroids Diabetes: from mechanical to treatment? The Netherlands Journal of Medicine 2014; 72:2.

8. Sudlow A, O'Connor HM, Narwani V, Swafe L, Dhatariya K. Assessing the prevalence of dexamethasone use in patients undergoing surgery, and subsequent glucose measurements: a retrospective cohort study. Pract Diab 2017; 34(4):117-121.

9. Montori VM, Velosa JA, Basu A et al. Posttrasplantation diabetes: A systematic review of the literature. Diabetes Care. 2002;25: 583-92.

10. Gunar T, Jan B, Goran S et al. Improvement survival in patients with insulin dependent diabetes mellitus and end-state diabetic nephropathy 10 years after combined pancreas and kidney transplantation1. Transplantation. 1999; 67:5: 645-648.

11. Umpierrez GE, Isaacs SD, Bazargan N, You X, Thaler LM, Kitabchi AE. Hyperglycemica: an independent marker of inhospital mortality in patients with undiagnosed diabetes. J Clin Endocrinol Metab.2002;87:978-982.

12. Ingle DJ. Production of glycosuria In normal rat by means of 17 -hydroxy11-dehydrocorticosterone. Endocrinology 1941; 29:649-52.

13. McMahonM, Gerich J, Rizza R. Effects of glucocorticoids on carbohydrate metabolism. Diabetes Metab Rev. 1988;4: 17-30.

14. Vegiopoulos A, Herzig S. Glucocorticoids, metabolism and metabolic diseases. Mol Cell Endocrinol. 2007; 275:43-61.

15. Van Raalte DH, Ouwens DM, Diamant M. Novel insights into glucocorticoidmediated diabetogenic effects: Towards expansion of therapeuthic options? Eur J Clin Invest. 2009; 39:81-93.

16. Corssmit EP, Romijn JA, Sauerwein HP. Regulation of glucose production with special attention to nonclassical regulatory mechanisms: A review. Metabolism 2014; 50(7):742-755.

17. Boden G. Gluconeogenesis and glycogenolysis in health and diabetes. J Investig Med 2004; 52(6):375-378.

18. Van de Werve G, Jeanrenaud B. Liver glycogen metabolism: An overview. Diabetes Metab Rev 1987; 3(1):47-78.

19. Gerich JE, Lorenzi M, Bier DM et al. Effects of physiologic levels of glucagon and growth hormone on human carbohydrate and lipid metabolism. Studies involving administration of exogenous hormone during suppression of endogenous hormone secretion with somatostatin. J Clin Invest 1976; 57(4):875-884.

20. Rizza RA, Mandarino LJ, Gerich JE. Dose-response characteristics for effects of insulin on production and utilization of glucose in man. Am J Physiol Endocrinol Metab 1981; 240(6):E630-E639.

21. Dungan KM, Braithwaite SS, Preiser J-C. Stress hyperglycaemia. Lancet 2009; 373(9677):1798-1807.

22. McCowen KC, Malhotra A, Bistrian BR. Stress-induced hyperglycemia. Crit Care Clin 2001; 17(1):107-124.

23. Stentz FB, Umpierrez GE, Cuervo R, Kitabchi AE. Proinflammatory cytokines, markers of cardiovascular risks, oxidative stress, and lipid peroxidation in patients with hyperglycemic crises. Diabetes 2004; 53(8):2079-2086.

24. Chaudhuri A, Umpierrez GE. Oxidative stress and inflammation in hyperglycemic crises and resolution with insulin: implications for the acute and chronic complications of hyperglycemia. J Diabetes Complications 2004; 26(4):257-258.

25. Reyes-Umpierrez D, Davis G, Cardona S et al. Inflammation and oxidative stress in cardiac surgery patients treated to intensive versus conservative glucose targets. J Clin Endocrinol Metab 2016; 102(1):309-315.
26. Riddle MC, et al. Standards of medical care in diabetes-2018. American diabetes association. The Journal of clinical and Applied Research and education. 2018;41; s144-151.

27. Moghissi ES, Korytkowski M, DiNardo M, et al.; American Association of Clinical Endocrinologists; American Diabetes Association. American Association of Clinical Endocrinologist and American Diabetes Association consensus statement on inpatients glycemic control. Diabetes Care 2009; 32: 1119-1131.

28. Institute of Medicine. Preventing Medication Errors. Aspden P, Wolcott J, Bootman JL, Cronewett LR, Eds. Washington, DC, The National Academies Press, 2007.

29. Wang YJ, Seggelke S, Hawkins RM, et al. Impact of glucose management team on outcomes of hospitalizaron in patients with type 2 diabetes admitted to the medical service. Endocr Pract 2016; 22:1401-1405.

30. International Hypoglycaemia Study Group. Glucose concentrations of less than $3.0 \mathrm{mmol} / \mathrm{L}(54 \mathrm{mg} / \mathrm{dL})$ should be reported in clinical trials: a join position statement of the American Diabetes Association and European Association for the Study of Diabetes. Diabetes Care 2017; 40: 155-157.

31. Clore J, Thurby-Hay L. Glucocorticoid-induced hyperglycemia. Endocr Pract 2009;15:469-74

32. Kwon S, Hermayer KL. Glucocorticoidinduced hyperglycemia. Am J Med Sci 2013;345: 274-277.

33. Burt MG, Drake SM, Aguilar-Loza NR, Esterman A, Stranks SN, Roberts GW Efficacy of a basal bolus insulin protocol to treat prednisolone-induced hyperglycaemia in hospitalised patients. Internal Medicine Journal. 2015

34. Trence DL. Management of patients on chronic glucocorticoid therapy: an endocrine perspective. Prim Care 2003; 30:593-605.

35. Campbell PJ, Gerich JE. Impact of obesity on insulin action in volunteers with normal glucose tolerance: demonstration of a threshold for the adverse effect of obesity. J Clin Endocrinol Metab 1990; 70:1114-18.

36. Maynard G, Wesorick DH, O'Malley C, Inzucchi SE; Society of Hospital Medicine Glycemic Contorl task Force. Subcutaneous insulin order sets and protocols: effective design and implementation strategies. J Hosp Med 2008; 3 (Suppl.): 29-41.

37. Corsino L, Dhatariya K, Umpierrez G. Management of diabetes and hyperglycemia in hospitalized patients. In Endotext [Internet]. Available from http:// www.ncbi.nlm.nih.gov/books/NBK279093/ Consultado el 28 de octubre de 2018.

38. Kwon S, Hermayer KL. Glucocorticoid-induced hyperglycemia. Am J Med Sci 2013; 345: 274-277.

39. Brady V, Thosani S, Zhou S, Bassett R, Busaidy NL, Lavis V. Safe and effective dosing of basal-bolus insulin in patients receiving high-dose steroids for hyper-cyclophosphamide, doxorubicin, vincristine, and dexamethasone chemotherapy. Diabetes Technol Ther 2014; 16: 874-879.

40. Xiao-xia L, Xiao-Ging Z, Qing M et al. Hyperglycemia induced by glucocorticoids in non-diabetic patients: A Meta-Analysis. Ann Nutr Metab. 2014; 65: 324-332.

41. Lhakani OJ, Kumar S, Triphati S, et al. Comparison of two protocols in the management of glucocorticoid-induced hyperglycemia among hospitalized patients. Indian Journal of Endocrinology and Metabolism. 2017;21,6:836-844

42. Umpierrez GE, Hellman R, Korytkowski MT, et al. Management of hyperglycemia in hospitalized patients in non-critical care setting: an endocrine society clinical practice guideline. J Clin Endocrinol Metab. 2012; 97:16-38.

43. Hawkins K, Donihi AC, Korytkowaski MT. Glycemic management in medica and surgical patients in the non-ICU settings. Curr Diab Rep 2013; 13:96-106.

44. Najman A, Andre K, Gorin NC: [Influence of comorbidities on decision caring of malignant haematological diseases]. Bull Cancer 2009; 96:563-570.

45. Paulsen $\emptyset$, Aass N, Kaasa S, et al. Do corticosteroids provide analgesic effects in cancer patients? A systematic literature reviews. J Pain Symptom Manage 2013; 46:96-105.

46. Roscoe JA, Heckler CE, Morrow GR, et al. Prevention of delayed nausea: A University of Rochester Cancer Center Community Clinical Oncology Program study of patients receiving chemotherapy. J Clin Oncol 2012; 30:3389-3395.

47. Brady V, Thosani S, Zhou S, Bassett R, Busaidy NL, Lavis V. Safe and effective dosing of basal-bolus insulin in patients receiving high-dose steroids for hyper-cyclophosphamide, doxorubicin, vincristine, and dexamethasone chemotherapy. Diabetes Technol Ther 2014; 16: 874-879. 